\title{
Bemerkungen zur operativen Behandlung innerer Incarceration.
}

\author{
Von Prof. Czerny.
}

Da ich in den oben mitgetheilten zwei Fällen von innerer Einklemmung die Laparotomie mit Beseitigung des Hindernisses versucht habe, könnte es den Anschein haben, als ob ich in allen Fällen von innerer Einklemmung der breiten Eröffnung der Bauchhöhle den Vorzug geben würde vor der Anlegung eines künstlichen Afters. Ich möchte diese Meinung nicht aufkommen lassen, weil ich der Ueberzeugung bin, dass wir trotz oder vielleicht grade wegen unserer grösseren Erfahrungen bei Bauchschnitten festhalten müssen, an gewissen Medicationen, wie sie eigentlich schon Nélaton und Tüngel aufgestellt haben bezüglich der operativen Behandlung dieser wichtigen Erkrankungen. Ich fühle mich um so mehr verpflichtet zu einer Meinungsäusserung, weil ich glaube, dass die jüngsten Autoren über diesen Gegenstand die Laparotomie allzusehr in den Vordergrund gestellt haben. Dass sie die ideal vollkommene Operation ist, kann ja nicht bezweifelt werden. Allein wer jemals bei stark tympanitischem Darme und prall gespannten Bauchdecken den Unterleib eröffnet hat, ohne dass durch Entfernung grösserer Geschwulst- oder Flüssigkeitsmassen Raum geschafft werden konnte, wird zugestehen, dass or froh war, als er die Därme wieder in die Bauchhöhle zurückgebracht und die Bauchwunde mit festzugeschnürten Nähten geschlossen hatte. Die Gefahren der langen Entblössung und Abkühlung, dann des mechanischen Insultes der geblähten, paralytischen Darmschlingen ist eine so grosse, dass man einen ohnehin durch Inanition, Erbrechen, Verdrängung des Zwerchfells und Athemnoth geschwächten Menschen diesem Versuche, das Hinderniss durch den Bauchschnitt aufzusuchen, nicht aussetzen sollte.

Ich möchte also ganz kategorisch die Indication aufstellen, dass bei der Diagnose einer inneren Incarceration die künstliche Afterbildung vorgenommen werden müsse, sobald der Leib trommelartig aufgetrieben und sowohlder Sitz 
als die Natur des Hindernisses zweifelhaft ist. Wenn unter diesen Umständen grosse Eingiessungen mit Wasser auch Gas, wenn die Magenpumpe keine natürliche Passage für Koth und Winde herbeigeführt haben, erscheint mir die künstliche Afterbildung ebenso dringend indicirt, wie die Tracheotomie bei gefahrdrohender Laryngostenose.

Die typische Stelle wird unter diesen Umständen immer die Nélaton'sche lleotomie sein müssen. Blos wenn man guten Grund hat, die Hindernisse im untersten Abschnitte des Dickdarmes zu vermuthen, wird man Colostomie machen dürfen. Ich empfehle die einzeitige Operation und möchte wie Tüngel rathen, die Fistel blos kleinfingerdick anzulegen, da sie ja blos ein Sicherheitsventil darstellen soll. Nach Eröffnung des Bauchfells wird dasselbe am besten mit der Hautwunde durch einige Seiden- oder Catgutnähte lippenförmig umwunden. Dadurch vermeidet man Bauchdeckenphlegmone und bekommt breite Berührungsflächen der Serosa. Dann wird die nächste geblähte Darmschlinge mit einem Hakenschieber gefasst, etwas in die Bauchwunde vorgezogen und mit einem dichten Kranz von Knopfnähten in der Bauchwunde befestigt. Man benützt dazu nur breite stark gekrümmte feine Nadeln und Seide oder Catgut. Dann wird in die Darmschlinge eine kleine Deffnung gemacht und der vorquellende Inhalt mit blauem Salicyl wasser oder $1: 5000$ Sublimatlösung abgespült. Wenn nichts mehr abfliesst, muss man mit Silberdraht und Nadeln, welche vom Darmlumen aus durch die Bauchdecken gestochen werden, das Darmrohr noch durch eine zweite Nahtreihe sicher befestigen und die Schleimhautenden lippenförmig mit der Hautwunde in Berührung bringen. Jodoformverband. Die Operation ist einfach, so wenig schmerzhaft dass sie selbst ohne Narkose gemacht werden kann und so gefahrlos, dass sie unmöglich die Situation verschlimmern kann. Jeder practische Arzt müsste dieselbe ausführen. Dass man selbst unter den schwierigsten Verhältnissen mit dieser Operation Frfolg erzielen kann, beweist mir folgender Fall aus meiner Praxis.

Am 5. December 1882 wurde ich nach Pforzheim gerufen zu einem 14jährigen Knaben (Eugen S.), der früher bis auf Neigung zu. Diarrhöen ¡mmer gesund war und am 1. Dec. nach einer reichlichen Mahlzeit (Gullasch 
mit Kartoffeln) plötzlich sehr heftige kolikartige Bauchschmerzen bekam. Vor 6 Wocben soll er ebenfalss einen leichten Kolikanfall gehabt haben. Am 2. Dec. trat Erbrechen von Speisen auf, ohne sich zu wiederholen. Der Leib wurde aufgetrieben, eine Entleerung wurde durch grosse Wassereingiessungen ohne Erfolg versucht. Die Schmerzen und Tympanitis nahmen enorm zu, so dass der Knabe fortwährend stöhnte und wimmerte, wenn er nicht im Morphiumdusel erhalten wurde. Am 3. Dec. hatten drei Aerzte die Wahrscheinlichkeitsdiagnose auf perforative Peritonitis gestellt und sahen den Fall als hoffnungslos an. Die Erscheinungen hatten noch zugenommen, als ich den Knaben am 5. Dec. Nachmittags sah. Durch den Hochstand des Zwerehfells war grosse Beengung und Athemnoth aufgetreten. Der Puls 112, regelmässig, die Temperatur normal. Der Unterleib colossal gespannt, sehr schmerzhaft, aber gegen Druck nicht sehr empfindlich. Keine abnorme Dämpfung und auch kein Tumor nachweisbar. Der allgemeine Kräftezustand wohl sehr reducirt, aber doch noch nicht ganz erschöpft.

Icb hielt eine innere Incarceration für wabrscheinlicher als eine perforative Peritonitis, weil die Erscheinungen bei einem vorher gesunden Knaben plötzlich aufgetreten waren und weil am 5. Tage nach einer Perforation entweder schon der Tod erfolgt sein oder doch eine circumscripte Peritonitis vorhanden sein müsste. Ich empfahl deshalb als letztes Rettungs- und Erleichterungsmittel die Anlegung einer Darmfistel an der Nélaton'schen Stelle. Nach Eröffnung des Bauchfells flossen Esslöffel voll trüben Serums heraus und es präsentirte sich eine mässig gespannte Dünndarmschlinge, welche einen dünnen fibrinösen Belag zeigte. Die Operation wurde in der oben skizzirten Weise vollendet. Nach der Eröffung des Darmrobres kamen wohl Flatus, aber kein Koth heraus. Erst am folgenden Tag gelang es- dew behandelnden Arzte durch Einführung eines dicken weichen Gummirohres flässigen Koth mit sichtlicher Erleichterung heranszubefördern. Der Leib ist weniger gespannt, das Allgemeinbefinden gut. Temp. 37. Puls 96. Am 7. Dec. ist der Leib ziemlich gefallen; der Patient behauptet, es seien Winde durch den After abgegangen. Die A thembeschwerden haben nachgelassen, der Urin wird spontan gelassen. Noch heftige Schmerzen um den Nabel herum. Champagner, Schleimsuppe, Thee werden gut vertragen.

9. Dec. Stublentleerung auf natürlichem Wege, darauf etwas Diarrhöe. Durch die Fistel geht dünner Koth in geringen Mengen ab. Nestle's Kindermehl.

15. Dee. Wegen der diarrhoisehen Stühle, welche übrigens niemals Blut enthielten, manchmal Narcotica. Die Entleerung aus der Fistel ist grangelb, dünnflüssig, manchmal chymusartig. Befinden gut. Taubenfleisch wird mit Appetit gegessen. Der Unterleib fühlt sich noch besonders in der Blasengegend ziemlich fest an und ist etwas aufgetrieben. Die Reconvalescenz langsam, Abmagerung hochgradig, etwas Decubitus.

28. Dec. Die Schmerzanfälle wiederholten sich noch öfters bis zum 26. Dec. In den letzten drei schmerzfreien Tagen hat er sich recht erholt und geniesst mit Vorliebe Fleischspeisen. Die Diarrhöe hat nachgelassen 
und aus der Fistel kommt ziemlich dicker Koth. Bei grossen Klystieren mit 1. 1 Wasser kam das Wasser aus der Fistel im Strahl herans. Die höchsten Temperaturen waren 38,2 .

Der Knabe erholte sich sehr rasch und sah bald besser aus, als vor der Operation, namentich war die Neigung zu Diarrhöen verschwunden. Eine kleine Bandage mit Pelotte konnte die Fistel vollkommen verschliessen. Ende October 1883 war die Fistel noch $1 \mathrm{~cm}$ im Durchmesser, belästigte ihn wenig. $Z$ wei Aetzungen mit dem Thermokauter hatten nur wenig Erfolg.

Am 6. August 1884 nähte ich die Fistel in der Klinik zu. Die Schleimhant wurde soweit zurückpräparirt, dass sie eingestülpt und ibre wunden Flächen mit Catgut genäht werden konnten. Darüber nähte ich die angefurchten Hantränder mit 3-4 Silberdrähten zu. In 8 Tagen war die Fistel gebeilt und der Patient ist seitdem so wobl geblieben, dass er sich zum Abiturientenexamen vorbereitet.

Ob es sich um eine Intassusception oder um eine Axsendrehung oder ein sonstiges Hinderniss gehandelt habe, wage ich nicht zu entscheiden. Genug, dass unter ganz verzweifelten Verhältnissen bei beginnender diffuser Peritonitis, weit entfernt von der klinischen Anstalt noch durch die Ileostomie ein vollkommener. Erfolg erzielt worden ist.

Ich möchte deshalb die Laparotomie und Aufsuchung der Hindernisse blos für diejenigen Fälle innerer Incarceration reserviren, in welchen die Kraft der Patienten noch gut erhalten sind, der Leib noch weich und nicht gespannt ist und wo man durch Palpation in der Narkose wenigstens den Ort der Hindernisse mit einiger Sicherheit feststellen kann. Dass dann der Bauchschnitt am besten an dieser Stelle vorgenommen werden soll, ist wohl selbstverständlich. 